Review

\title{
Fundamentals of Diatomic Molecular Spectroscopy
}

\author{
Christian G. Parigger $1, *, \mathbb{D}$
}

1 Physics and Astronomy Department, University of Tennessee, University of Tennessee Space Institute, Center for Laser Applications,411 B.H. Goethert Parkway, Tullahoma, TN 37388-9700, USA; cparigge@tennessee.edu * Correspondence: cparigge@tennessee.edu; Tel.: +1 (931) 8415690

\begin{abstract}
The interpretation of optical spectra requires thorough comprehension of quantum mechanics, especially understanding the concept of angular momentum operators. Suppose now that a transformation from laboratory-fixed to molecule-attached coordinates, by invoking the correspondence principle, induces reversed angular momentum operator identities. However, the foundations of quantum mechanics and the mathematical implementation of specific symmetries assert that reversal of motion or time reversal includes complex conjugation as part of anti-unitary operation. Quantum theory contraindicates sign changes of the fundamental angular momentum algebra. Reversed angular momentum sign changes are of heuristic nature and are actually not needed in analysis of diatomic spectra. This work addresses sustenance of usual angular momentum theory, including presentation of straightforward proofs leading to falsification of the occurrence of reversed angular momentum identities. This review also summarises aspects of a consistent implementation of quantum mechanics for spectroscopy with selected diatomic molecules of interest in astrophysics and in engineering applications.
\end{abstract}

Keywords: Foundations of Quantum Mechanics; Molecular Spectroscopy; Diatomic Molecules; Symmetry Transformations; Optical Emission Spectroscopy; Astrophysics

\section{Introduction}

Identification of diatomic molecular spectra necessitates a clear description of angular momentum (AM) in order to demarcate the various features that comprise optical fingerprints. Quantum mechanics theory (QMT) asserts that not all three components of AM can be measured simultaneously, usually the total AM and one projection of the total AM describe upper and lower states of molecular transitions. The components of AM are formally described by a set of operator equation.

Classical mechanics (CM) description and associated quantization of the asymmetric top [1] suggests occurrence of commutator relations with different signs when computing momenta with respect to the principal axes of inertia. In other words, a laboratory-fixed system shows standard AM commutators, but with respect to the molecule-attached coordinate system there is a sign change that carries the name "reversed" internal AM [2]. The derivation by Klein in 1929 [1] is based on the correspondence principle that in essence emphasizes that QMT reproduces classical physics in the limit of large quantum numbers. From a CM point of view, reversal of motion occurs when transforming from a lab-fixed to a molecule-attached coordinate system, akin to experience of motion reversal when jumping onto a moving merry-go-around. However, reversal of motion in quantum mechanics $(\mathrm{QM})$ is described by an anti-unitary transformation, requiring sign change and complex conjugation. The reversed internal AM concept [2] and applications actually are communicated and applied in analysis of molecular spectra by Van Vleck in 1951 in his review article on coupling angular momenta, i.e., AM referred to axes mounted on the molecule adheres to opposite-sign commutator algebra. This evolved into so-called reversed angular momentum (RAM) concepts for prediction of molecular spectra.

However, orthodox or classic QM abides by strict mathematical rules associated with the theory. Use of RAM techniques is contraindicated, especially since Nöther-type symmetry transformation [3] sustain the standard commutator relations, viz. reversal of

motion is an anti-unitary transformation, just like in the Schrödinger wave equation that is

Publisher's Note: MDPI stays neutra with regard to jurisdictional claims in published maps and institutional affiliations. 
invariant with respect to motion-reversal or time-reversal due to anti-unitary operation, as expected. It is important to recognize that a transformation from laboratory-fixed to molecular-attached coordinates within standard QM does not condone anomalous AM operator identities.

This work communicates proofs that the quantum-mechanic AM equations remain the same in a transition from laboratory-fixed to molecular-attached coordinates. Methods that invoke RAM for the prediction of molecular spectra are misleading. Application of standard QM establishes within the concept of line strengths [4] consistent computation of diatomic spectra [5], examples include hydroxyl, cyanide and diatomic carbon spectra [6].

\section{Materials and Methods}

The premise of this article is Oscar Klein's work [1] "Zur Frage der Quantelung des asymmetrischen Kreisels" or "On the question of the quantization of the asymmetric top." This particular work is in German without an available translation, the essential contents are in the Einleitung, viz. introduction, and on the page following the introduction. Klein's paper reflects the initial argumentation of the RAM method, summarized below.

The purpose of the 1929 work is, as O. Klein writes, to reduce quantization of the asymmetric top to simple algebra for the components of the angular momentum "... that were developed by Dirac [7] and as well by Born, Heisenberg and Jordan [8]." For a solid body, the main moment of inertia are labeled as $A, B$, and $C$, the angular momenta are labeled $P, Q, R$, and one finds the CM energy of rotation, $E$,

$$
E=\frac{1}{2}\left(\frac{P^{2}}{A}+\frac{Q^{2}}{B}+\frac{R^{2}}{C}\right)
$$

or perhaps with convenient notation, using for operators $\tilde{J}_{1}=P, \tilde{J}_{2}=Q, \tilde{J}_{3}=R$, where the tilde-symbol indicates that angular momenta (that would be AM operators in QM) are referred to the main axis of the ellipse of inertia (or in molecules, referred to molecular-fixed coordinates), and for moment of inertia $I_{1}=A, I_{2}=B, I_{3}=C$,

$$
E=\frac{1}{2} \sum_{k=1}^{k=3} \frac{1}{I_{k}} \tilde{J}_{k}
$$

Subsequently, O. Klein writes that $P, Q, R$ can be understood to describe matrices satisfying QM equations of motion, with $i=\sqrt{-1}$ and using the standard $\hbar$ for Planck's constant divided by $2 \pi$,

$$
\frac{d P}{d t}=\frac{i}{\hbar}(E P-P E), \quad \frac{d Q}{d t}=\frac{i}{\hbar}(E Q-Q E), \quad \frac{d R}{d t}=\frac{i}{\hbar}(E R-R E) .
$$

In terms of operators, using the Hamilton operator $\mathcal{H}$ instead of $E$ and writing the equation in the Heisenberg-picture for an abstract observable (operator), $\mathcal{O}$, without explicit timedependence of the observable, i.e., $\frac{\partial \mathcal{O}}{\partial t}=0$, and using the commutator $[\mathcal{H}, \mathcal{O}]=\mathcal{H O}-$ $\mathcal{O H}$

$$
\frac{d \mathcal{O}}{d t}=\frac{i}{\hbar}[\mathcal{H}, \mathcal{O}]+\frac{\partial \mathcal{O}}{\partial t} .
$$

The hypothesis of O. Klein comprises the requirement of utilizing Eq. (3) in Eq. (1). Consequently, $\mathrm{O}$. Klein assumes commutator relations for $P, Q, R$,

$$
i \hbar P=R Q-Q R, \quad i \hbar Q=P R-R P, \quad i \hbar R=Q P-P Q,
$$

or using abbreviated nomenclature and the Levi-Civita symbol, with $\varepsilon_{k l m}=1$ for even permutations, and $\varepsilon_{k l m}=-1$ for odd ones, otherwise $\varepsilon_{k l m}=0$ for identical indices, $k, l, m=1,2,3$,

$$
\left[\tilde{J}_{k}, \tilde{J}_{l}\right]=-i \hbar \varepsilon_{k l m} \tilde{J}_{m}
$$


With the commutator relations in Eqs. (5), the correspondence principle leads to the equations of motion, and as O. Klein writes "... as we overlook occurrence of the action-quant ...," viz. overlook $\hbar$. Further, O. Klein remarks that Eqs. (5) differ only by the sign of $i$ from the well known quantum-mechanical commutators for a laboratory-fixed system. Clearly, writing Eqs. (5) in the compact form of Eq. (6), highlights the minus sign that differs from the standard equations of AM operators $J_{k}, k=1,2,3$,

$$
\left[J_{k}, J_{l}\right]=i \hbar \varepsilon_{k l m} J_{m} \text {. }
$$

The minus sign in Eq. (6) is labeled "anomalous" by some authors, e.g., J. Van Vleck [2], but there is no justification for the anomalous minus sign to occur within QMT. Usually one considers right hand systems, so Eq. (7) is termed as the standard quantum-mechanic AM operator identity. Sustenance of RAM concepts may appear convenient, even calling the negative sign an "anomaly" but without QMT support. In scientific approach and in spite of the initial success in explaining spectra within various approximations, one usually avoids starting with an "anomaly" and/or inaccurate presuppositions that are readily falsified [9]. However, several textbooks and works continue support of RAM in the theory of molecular spectra [10-22], in spite of obvious falsification by QMT. This work emphasizes that there is no need to resort to RAM "cook book" [22] methods.

The methods in this work utilize standard QMT $[23,24]$ and standard mathematical methods [25] showing that there is no sign change of the standard commutator relations when transforming from a laboratory-fixed to a molecule-attached coordinate system. Consistent application of standard AM algebra in the establishment of computed spectra yield nice agreement with laboratory experimental results [5] and agreement in analysis of astrophysical $\mathrm{C}_{2}$ Swan data from the white dwarf Procyon B [5], including agreement in comparisons with computed spectra that are obtained with other molecular fitting programs such as PGOPHER [26].

Methods for measurement of optical emission signals from diatomic molecules are comprised of standard molecular spectroscopy experimental arrangements such as in laser-induced plasma or breakdown spectroscopy [27-34], encountered as well in stellar plasma physics or astrophysics to name other areas of interest.

\section{Results}

\subsection{Angular Momentum Commutators}

The invariance of standard QMT commutator relations, see Eq. (7), is communicated in this section.

\subsubsection{Invariance for unitary transformations}

Application unitary transformation, viz. transforming from one coordinate system to another, leaves the AM commutator relations invariant [35]. A unitary transformation operator, $U$, acting on an operator $\mathcal{O} \longrightarrow \mathcal{O}^{\prime}$, with $U^{+}=U^{-1}$, is defined by

$$
\mathcal{O}^{\prime}=U \mathcal{O} U^{\dagger} \quad \text { or } \quad \mathcal{O}=U^{\dagger} \mathcal{O}^{\prime} U
$$

Consequently,

$$
U^{\dagger} J_{k}^{\prime} U U^{\dagger} J_{l}^{\prime} U-U^{\dagger} J_{l}^{\prime} U U^{\dagger} J_{k}^{\prime} U=U^{\dagger} J_{k}^{\prime} J_{l}^{\prime} U-U^{\dagger} J_{l}^{\prime} J_{k}^{\prime} U=i \varepsilon_{k l m} U^{\dagger} J_{m}^{\prime} U,
$$

preserving the quantum-mechanic AM commutators, see Eq. (7). For example, the Euler rotation matrix is easily demonstrated to be unitary [6]. In other words, there is no anomaly when going from a laboratory-fixed to a molecule-attached coordinate system.

\subsubsection{Invariance for time reversal or reversal of motion}

Time reversal or reversal of motion in QMT requires sign changes of the operators and complex conjugation, leaving the QMT commutators invariant, 


$$
\left[J_{k}, J_{l}\right]=i \varepsilon_{k l m} J_{m} \longleftrightarrow\left[\left(-J_{k}\right),\left(-J_{l}\right)\right]=(-i) \varepsilon_{k l m}\left(-J_{m}\right) .
$$

$\mathrm{CM}$ would indicate a reversal of motion when going from a laboratory-fixed to a molecularfixed coordinate system, however, reversal of motion requires complex conjugation due to the anti-unitary requirement. In other words, the sign is preserved. QMT so-to-speak opposes the hypothesis by O. Klein.

The invariance regarding time reversal or reversal of motion of course also would apply to the abstract form of the tim-dependent Schrödinger equation,

$$
i \hbar \frac{\partial}{\partial t} \psi=\mathcal{H} \psi \quad \longleftrightarrow \quad(-i) \hbar \frac{\partial}{\partial(-t)} \psi=\mathcal{H} \psi,
$$

where $\psi$ describes an abstract vector in Hilbert space, and $\mathcal{H}$ is a Hamiltonian. Changing time $t \longrightarrow-t$ and applying conjugate complex of $i$ preserves the left hand side of the equation. For example for a free particle of mass $m$ and momentum $\mathcal{P}$, the Hamiltonian is $\mathcal{H}=\mathcal{P}^{2} / 2 m$, and the form of Schrödinger's equation is preserved.

Equally, the operator equation in the Heisenberg picture, see Eq. (4), preserves form under time reversal or reversal of motion,

$$
\frac{d \mathcal{O}}{d t}=\frac{i}{\hbar}[\mathcal{H}, \mathcal{O}]+\frac{\partial \mathcal{O}}{\partial t} \quad \longleftrightarrow \quad \frac{d(-\mathcal{O})}{d(-t)}=\frac{(-i)}{\hbar}[\mathcal{H},(-\mathcal{O})]+\frac{\partial(-\mathcal{O})}{\partial(-t)}
$$

A change of sign for the operators and complex conjugation leaves the equation invariant. The mentioned symmetry can also be associated with usual Nöther symmetries [3].

\subsection{Diatomic wave function}

For diatomic molecules, symmetry properties allow one to invoke simplifications when evaluating the laboratory wave-function in terms of rotated coordinates [5]. For internuclear geometry, the spherical polar coordinates are $r, \phi, \theta$, and one (arbitrary) electron is described by cylindrical coordinates $\rho, \chi, \zeta$. For coordinate rotation one uses Euler angles $\alpha, \beta, \gamma$, and without loss of generality on can choose $\alpha=\phi, \beta=\theta, \chi=\gamma$ [5]. The result is the Wigner-Witmer eigenfunction (WWE) for diatomic molecules [36],

$$
\left\langle\rho, \zeta, \chi, \mathbf{r}_{2}, \ldots, \mathbf{r}_{N}, r, \theta, \phi \mid n v J M\right\rangle=\sum_{\Omega=-J}^{J}\left\langle\rho, \zeta, \mathbf{r}_{2}^{\prime}, \ldots, \mathbf{r}_{N}^{\prime}, r \mid n v\right\rangle D_{M \Omega}^{J^{*}}(\phi, \theta, \chi) .
$$

The usual total AM quantum numbers are $J$ and $M$, and the electronic-vibrational eigenfunction is explicitly written by extracting $v$ from the collection of quantum numbers, $n$. The WWE exactly separates $\phi, \theta, \chi$. The quantum numbers $J, M, \Omega$ refer to the total AM. The sum over $J$ in Eq. (13) originates from the usual abstract transformation,

$$
|J M\rangle=\sum_{\Omega=-J}^{J}|J \Omega\rangle\langle J \Omega \mid J M\rangle,
$$

where $\Omega$ is the magnetic quantum number along the rotated, or new $z^{\prime}$-axis. The sum in Eq. (14) ensures that the quantum numbers for total AM are $J$ and $M$. In Hund's case $a$ [37], $\Omega$ describes the projection of the total AM, within L-S coupling. Hund's case $a$ eigenfunctions form a basis, therefore, from a computational point of view, these eigenfunctions form a complete (sufficient) set. In various approximate descriptions and for specific diatomic molecules, it may be desirable to use other Hund cases.

From the rotation operator $\mathcal{R}(\alpha, \beta, \gamma)$, with the Euler angles $\alpha, \beta, \gamma$, one finds for D-matrix elements,

$$
D_{M \Omega}^{J^{*}}(\alpha, \beta, \gamma)=\langle J M|\mathcal{R}(\alpha, \beta, \gamma)| J \Omega\rangle^{*}
$$


D-matrices are the usual mathematical tool for transformation from one basis to another, but the D-matrix cannot represent an eigenfunction due to presence of two magnetic quantum numbers $M$ and $\Omega$, so the sum over $J$ is needed in the transformed coordinates.

Diatomic spectra composed of line positions and line strengths are based on WWE [5] instead of eigenfunctions used for the Born-Oppenheimer approximation. Extensive experimental studies confirm agreement of computed spectra with measured emission spectra from laser-induced optical plasma [5].

\subsection{Computed and measured diatomic spectra}

\subsubsection{Computed spectra of $\mathrm{OH}$ and $\mathrm{C}_{2}$}

Typical spectra of some diatomic molecules of general interest are presented. Figure 1 illustrates $\mathrm{OH}$ molecular spectra for different spectral resolutions. Figures 2, 3 show computed $\mathrm{C}_{2}$ Swan spectra for the vibrational sequences $\Delta v=-1,+1$. The details for the computation, line strength data for $C_{2}$ Swan bands, and programs are published [38]. The published program package [38] also includes a worked high-temperature cyanide example, the Boltzmann equilibrium spectrum program (BESP) for computation of equilibrium spectra and the Nelder-Mead temperature (NMT) routine that utilizes a non-linear fitting algorithm. The $\mathrm{OH}$ line strength data have been made available recently [39].

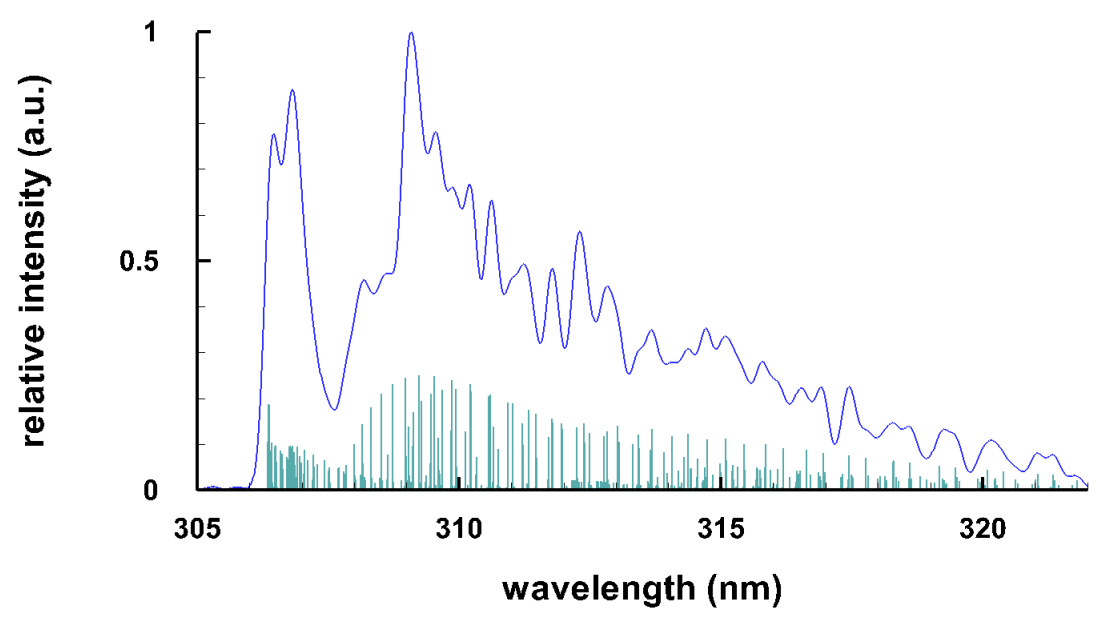

Figure 1. Computed spectrum of the $A^{2} \Sigma \rightarrow X^{2} \Pi$ uv Band of $\mathrm{OH}, \mathrm{T}=4 \mathrm{kK}$, (top) spectral resolutions of $\Delta \lambda=0.32 \mathrm{~nm}\left(\Delta \tilde{v}=32 \mathrm{~cm}^{-1}\right)$ and (bottom) idealized resolution for the stick spectrum $\Delta \lambda=0.002$ $\mathrm{nm}\left(\Delta \tilde{v}=0.2 \mathrm{~cm}^{-1}\right)$ of the $\Delta v=0$ sequence [6]. 


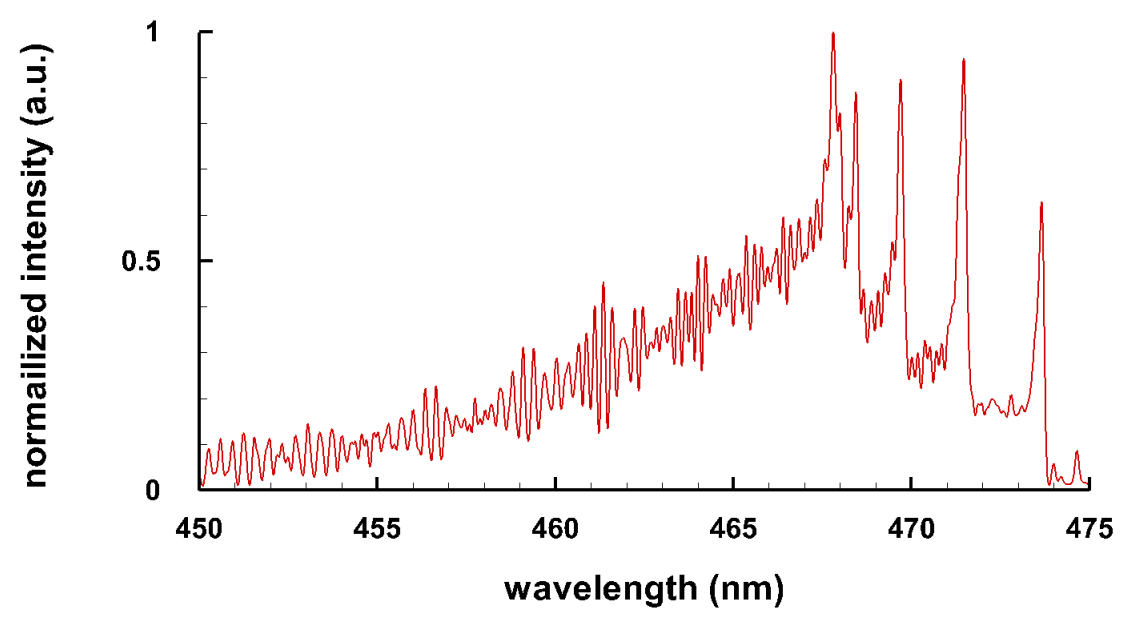

Figure 2. $\mathrm{C}_{2}$ Swan $d^{3} \Pi_{g} \rightarrow a^{3} \Pi_{u}$ band $\Delta v=-1$ sequence, $\mathrm{T}=8 \mathrm{kK}, \Delta \lambda=0.13 \mathrm{~nm}\left(\Delta \tilde{v}=6 \mathrm{~cm}^{-1}\right)$ [6].

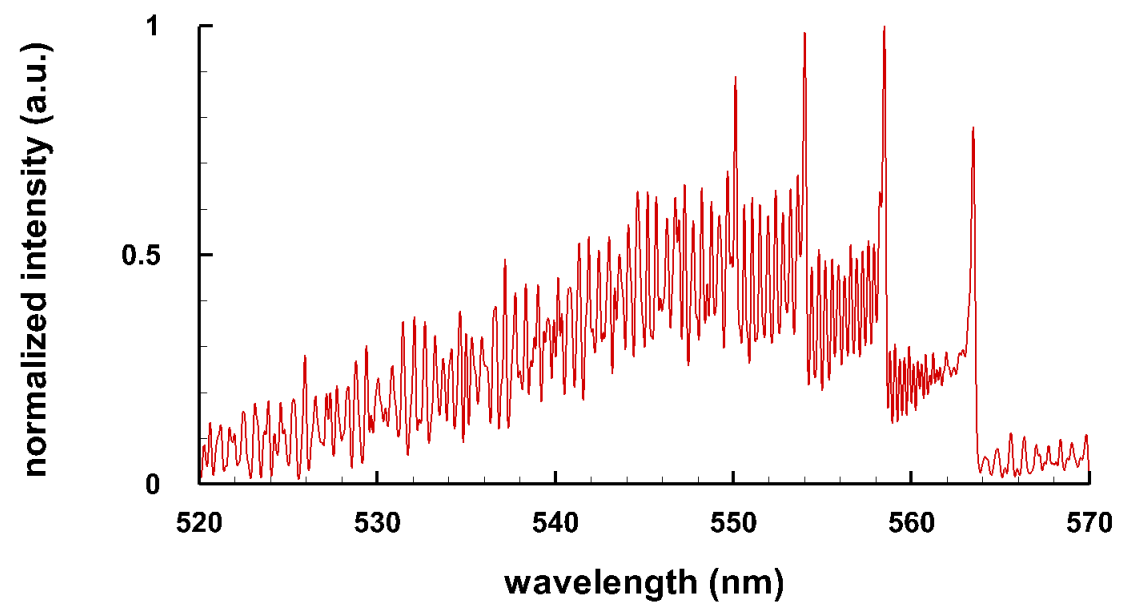

Figure 3. $C_{2}$ Swan $d^{3} \Pi_{g} \rightarrow a^{3} \Pi_{u}$ band $\Delta v=+1$ sequence, $T=8 \mathrm{kK}, \Delta \lambda=0.18 \mathrm{~nm}\left(\Delta \tilde{v}=6 \mathrm{~cm}^{-1}\right)$ [6].

\subsubsection{Comparisons with measured spectra}

Various reported studies of plasma spectra, including astrophysics plasma, and of molecular laser-induced breakdown spectroscopy [38-41] illustrate nice comparisons of recorded and of computed diatomic spectra.

\section{Discussion}

Angular momentum operators are well defined in quantum mechanics theory, including the fact that there is an inherent limit in measurement of its components. Another way of formulating this could be: There are only two quantum numbers needed for description of angular momentum, usually the total angular momentum and its projection onto a quantization axis. The use of the correspondence principle to ensure compatibility with classical mechanics equations of motion brings about ad hoc hypothesis of a negative sign for the commutators. Subsequent application of reversed angular momentum coupling occurs in the description of molecules that includes modeling of vector-operators as vectors. 
However, quantum mechanics theory already ensures how to mathematically describe angular momentum, not supporting heuristic conclusions involving reversed angular momentum concepts, nor occurrence of more than two quantum numbers for the total angular momentum of diatomic molecules. Consistent application of standard quantum mechanics theory is preferred, including avoidance of a priori use of separating electronic, vibrational, rotational wave functions. Subsequent to implementation of diatomic molecular symmetries, line strengths as function of wavelength are in agreement with results from optical emission spectroscopy. The computed and fitted diatomic spectra nicely match within reasonable error bars, but without invoking heuristic selection rules that may be affected by initial approximations or by spurious use of reversal of angular momentum.

Funding: This research received no specific grant-number external funding.

Acknowledgments: The author acknowledges support in part the State of Tennessee funded Center for Laser Applications at the University of Tennessee Space Institute.

Conflicts of Interest: The author declares no conflict of interest. The funders had no role in the design of the study; in the collection, analyses, or interpretation of data; in the writing of the manuscript, or in the decision to publish the results.

\section{Abbreviations}

The following abbreviations are used in this manuscript:

$\begin{array}{ll}\text { AM } & \text { Angular Momentum } \\ \text { BESP } & \text { Boltzmann Equilibrium Spectrum Program } \\ \text { CM } & \text { Classical Mechanics } \\ \text { NMT } & \text { Nelder-Mead Temperature } \\ \text { PGOPHER } & \begin{array}{l}\text { Program for simulating rotational, vibrational and electronic spectra, or } \\ \text { "Program Gopher" }\end{array} \\ \text { QM } & \text { Quantum Mechanics } \\ \text { QMT } & \text { Quantum Mechanics Theory } \\ \text { RAM } & \text { Reversed Angular Momentum } \\ \text { WWE } & \text { Wigner-Witmer Eigenfunction }\end{array}$

\section{References}

1. Klein, O. Zur Frage der Quantelung des asymmetrischen Kreisels. Z. Physik 1929, 58, 730-734.

2. Van Vleck, J.H. The Coupling of Angular Momentum Vectors in Molecules. Rev. Mod. Phys. 1951, 23, $213-227$.

3. Nöther, E. Invariante Variationsprobleme. Nachr. D. König. Gesellsch. D. Wiss., Göttingen 1918, 918 235-257; Invariant variation problems. Transp. Theory Stat. Phys. 1971, 1, 183-207.

4. Condon, E.U.; Shortley, G. The Theory of Atomic Spectra; Cambridge Univ. Press: Cambridge, UK, 1953.

5. Parigger, C.G.; Hornkohl, J.O. Quantum Mechanics of the Diatomic Molecule with Applications; IOP Publishing: Bristol, UK, 2020.

6. Parigger, C.G.; Hornkohl, J.O. Diatomic Molecular Spectroscopy with Standard and Anomalous Commutators. Int. Rev. At. Mol. Phys. 2010, 1, 25-43.

7. Dirac, P.A.M. The Elimination of Nodes in Quantum Mechanics. Proc. Roy. Soc. London A 1926, 111, $281-305$.

8. Heisenberg, W.; Born, M.; Jordan P. Zur Quantenmechanik II. Z. Physik 1926, 35, 557-615.

9. Popper, K. The Logic and Evolution of Scientific Theory. In: All Life is Problem Solving; Routledge: London, UK, 1999; Chap. 1, 3-22.

10. Brown, J.; Carrington, A. Rotational Spectroscopy of Diatomic Molecules; Cambridge Univ. Press: Cambridge, UK, 2003.

11. Lefebvre-Brion, H.; Field, R.W. The Spectra and Dynamics of Diatomic Molecules; Elsevier: Amsterdam, NL, 2004.

12. Bunker, P.R.; Jensen, P. Fundamentals of Molecular Spectroscopy; IOP Publishing: Bristol, UK, 2005.

13. Gottfried, K. Quantum Mechanics; Addison-Wesley: Reading, UK, 1989.

14. Baym, G. Lectures on Quantum Mechanics; Benjamin/Cummings: Reading, UK, 1969.

15. Shore, B.W.; Menzel, D.H. Principles of Atomic Spectra; Addison-Wesley: Reading, UK, 1968.

16. Judd, B.R. Angular Momentum Theory for Diatomic Molecules; Academic Press: New York, NY, USA, 1975.

17. Mizushima, M. The Theory of Rotating Diatomic Molecules; John Wiley \& Sons: New York, NY, USA, 1975.

18. Kovacs, I. Rotational Structure in the Spectra of Diatomic Molecules; Elsevier: New York, NY, USA 1969.

19. Hougen, J.T. The Calculation of Rotational Energy Levels and Rotational Line Intensities in Diatomic Molecules; N.B.S. Monograph 115, U.S. Government Printing Office: Washington, D.C., USA, 1970).

20. Kroto, H.W. Molecular Rotation Spectra; Dover: New York, NY, USA, 1992. 
21. Carrington, A.; Levy, D.H.; Miller, T.A. Electron Resonance of Gaseous Diatomic Molecules. Adv. Chem. Phys. 1970, 18, 149-248.

22. Freed, K.F. Theory of the Hyperfine Structure of Molecules: Application to ${ }^{3} \Pi$ States of Diatomic Molecules Intermediate between Hund's Cases (a) and (b).J. Chem. Phys. 1966, 45, 4214-4241.

23. Cohen-Tannoudji C.; Diu B.; Laloe, F. Quantum Mechanics, Volume 1: Basic Concepts, Tools, and Application, 2nd Edition; Wiley-VCH: Weinheim, DE, 2019.

24. Cohen-Tannoudji C.; Diu B.; Laloe, F. Quantum Mechanics, Volume 2: Angular Momentum, Spin, and Approximation Methds, 2nd Edition; Wiley-VCH: Weinheim, DE, 2019.

25. Arfken, G.B.; Weber, H.J.; Harris, F.E. Mathematical Methods for Physicists, A comprehensive Guide, Seventh Edition; Academic Press: New York, NY, USA, 2012.

26. Western, C.M. PGOPHER: A program for simulating rotational, vibrational and electronic spectra. J. Quant. Spectrosc. Radiat. Transfer 2017, 186, 221-242.

27. Kunze, H.-J. Introduction to Plasma Spectroscopy; Springer: Heidelberg, DE, 2009.

28. Demtröder, W. Laser Spectroscopy 1: Basic Principles, 5th Edition: Springer: Heidelberg, DE, 2014.

29. Demtröder, W. Laser Spectroscopy 2: Experimental Techniques, 5th Edition: Springer: Heidelberg, DE, 2015.

30. Hertel, I.V.; Schulz C.-P. Atoms, Molecules and Optical Physics 1, Atoms and Spectroscopy; Springer: Heidelberg, DE, 2015.

31. Hertel, I.V.; Schulz C.-P. Atoms, Molecules and Optical Physics 2, Molecules and Photons - Spectroscopy and Collisions; Springer: Heidelberg, DE, 2015.

32. Cremers, D.E.; Radziemski, L.J. Handbook of Laser-Induced Breakdown Spectroscopy; Wiley: New York, NY, USA, 2006.

33. Miziolek, A.W.; Palleschi, V.; Schechter, I. (Eds.) Laser Induced Breakdown Spectroscopy; Cambridge Univ. Press: New York, NY, 2006.

34. Singh, J.P.: Thakur, S.N., Eds. Laser-Induced Breakdown Spectroscopy, 2nd Edition; Elsevier: New York, NY, USA, 2020.

35. Davydov, A.S. Quantum Mechanics, Pergamon Press: Oxford, UK, 1965.

36. Wigner, E.; Witmer, E.E. Über die Struktur der zweiatomigen Molekelspectren nach der Quantenmechanik. Z. Physik 1928, 51, 859-886; Hettema, H. On the Structure of the Spectra of Two-Atomic Molecules According to Quantum Mechanics. In: Quantum Chemistry: Classic Scientific Papers; World Scientific: Singapore, SG, 2000, 287-311.

37. Bransden, B.H.; Joachain, C.J. Physics of Atoms and Molecules, second edition; Prentice Hall: Essex, UK, 2003.

38. Parigger, C.G.; Woods, A.C.; Surmick, D.M.; Gautam, G.; Witte, M.J.; Hornkohl, J.O. Computation of diatomic molecular spectra for selected transitions of aluminum monoxide, cyanide, diatomic carbon, and titanium monoxide. Spectrochim. Acta Part B: At. Spectrosc. 2015, 107, 132-138.

39. Parigger, C.G.; Helstern, C.M; Jordan, B.S.; Surmick, D.M.; Splinter, R. Laser-Plasma Spectroscopy of Hydroxyl with Applications. Molecules 2020, 25, 988, 18 pp.

40. Parigger, C.G.; Surmick, D.M.; Helstern, C.M.; Gautam G.; Bol'shakov, A.A.; Russo, R.E. Molecular laser-induced breakdown spectroscopy. In Laser-Induced Breakdown Spectroscopy, Second Edition; Eds. Singh, J.P.; Thakur, S.N.; Elsevier: Amsterdam, NL, 2020; Chap. 7, 167-209.

41. Parigger, C.G.; Helstern, C.M; Jordan, B.S.; Surmick, D.M.; Splinter, R. Laser-Plasma Spatiotemporal Cyanide Spectroscopy and Applications. Molecules 2020, 25, 615, 13 pp. 